\title{
Probability Estimation of Change Maps Using Spectral Similarity ${ }^{+}$
}

\author{
Hamid Jafarzadeh and Mahdi Hasanlou * \\ School of Surveying and Geospatial Engineering, College of Engineering, University of Tehran, 1417466191 \\ Tehran, Iran; h.jafarzadeh73@ut.ac.ir \\ * Correspondence: hasanlou@ut.ac.ir; Tel.: +98-21-6111-4525 \\ + Presented at the 3rd International Electronic Conference on Remote Sensing, 22 May-5 June 2019; \\ Available Online: https://sciforum.net/conference/ecrs-3.
}

Published: 4 June 2019

\begin{abstract}
Change Detection (CD), which is a process of identifying changes that have occurred in a geographical area over the time, plays a key role in many applications, including assessing natural disasters, monitoring crops, and managing water resources. In the past decades, many CD techniques have been proposed. Hence, evaluating and analyzing of the probability of changes and interpreting them is an essential task which leads to better management of natural resources and the prevention of disasters. For this purpose, we adopted an approach to estimate the probability of changes that have occurred in the image scene. Based on this approach, changed pixels are categorized and labeled as probabilities (in percentage format). In this paper, the proposed framework consists of the following four steps. Firstly, this research produces a final binary change map (BCM) through combining the results of some of popular binary CD methods that have been proposed in the literature. Then an unmixing process is adopted and in the next step the spectral similarity of pixels is calculated in the abundance maps of endmembers. A measurement of spectral similarity identifies the finer spectral differences between the two hyperspectral images (HSIs). Finally, spectral similarity values are masked by the final BCM resulting in a probability map of changes. The experimental results verified that the method is able to obtain good results and may be well suited for hyperspectral CD applications.
\end{abstract}

Keywords: Change Detection; hyperspectral; probability map; spectral similarity

\section{Introduction}

Change Detection (CD) is a fundamental and challenging subject of research, including remote sensing (RS), monitoring and surveillance, civil engineering, mechanical engineering, medical diagnosis, etc. [1,2]. In RS applications, CD is defined as the process of detecting the variations of materials in a given image scene, due to time or as a result of a significant event (such as a natural hazard) or natural metamorphoses, by analyzing co-registered multi-temporal images of the same geographical area acquired at different times [3]. The RS-CD has a wide range of practical applications in many fields, such as disaster assessment, studies on urban sprawl, land cover monitoring, environmental monitoring, and ecosystem monitoring [3,4]. The main principle for utilizing RS data for CD is that changes in the object of interest will alter the spectral behavior (reflectance value) that is separable from changes caused by factors such as atmospheric conditions, illumination and viewing angles, soil moistures, etc. [1,5].

The Earth's surface undergoes significant changes over time due to influences originating from the increasing urbanization and human population [1,6]. In addition, the demand for natural resources has been increasing, which leads to side effects on land cover and land use [1]. Hence, an 
analysis of the changes by the RS tools is necessary for the better management of natural resources and to help prevent disasters [6]. In addition, for planning purposes, estimating the probability (also can be defined as intensity) of changes is necessary.

In the past decades many binary $\mathrm{CD}$ techniques have been proposed for discriminating changed and no-changed pixels [6]. Estimating, evaluating, analyzing, and interpreting the probability of changes that have occurred in the image scene is an essential task which leads to better monitoring of variations in the study areas. The aim of this paper is to suggest a solution to produce a probability map of changes. For this purpose, we adopted an approach to estimate the probability of occurrence of detected changes using spectral similarities. Based on this approach, changes' probability map is produced, and pixels are categorized and labeled as percentage of probabilities.

\section{Experiments}

\subsection{Study Area and Data Set}

In this work, we utilized widely used real-world hyperspectral images (HSIs) to evaluate the performance of the proposed method. This data set is a reference and benchmark data set that has been used previously in many hyperspectral CD approaches [6], and can be found online (http://rslab.ut.ac.ir). Figure 1 shows the case study area that covers an irrigated agricultural field in the city of Hermiston in Umatilla County, Oregon, USA. The size of employed hyperspectral data set is $307 \times 241$ pixels. The data were acquired on May 1, 2004, and May 8, 2007. The main changes between the acquisition dates relate to land cover changes of agricultural fields.

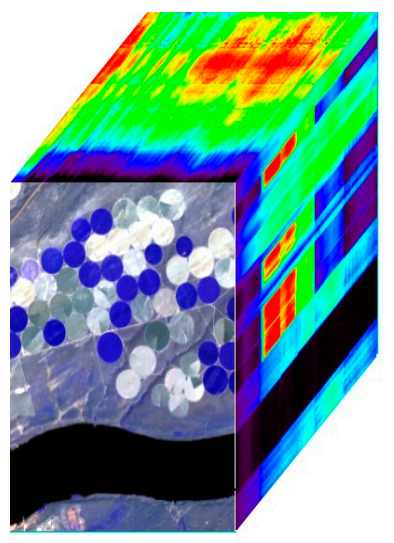

(a)

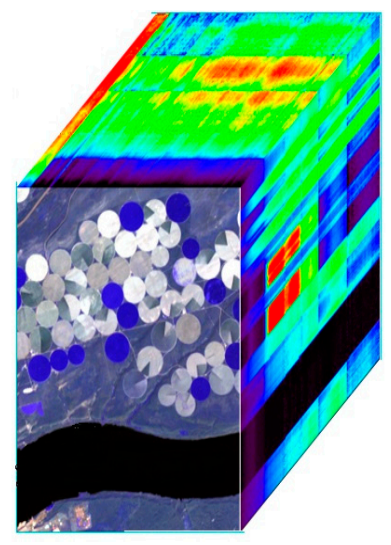

(b)

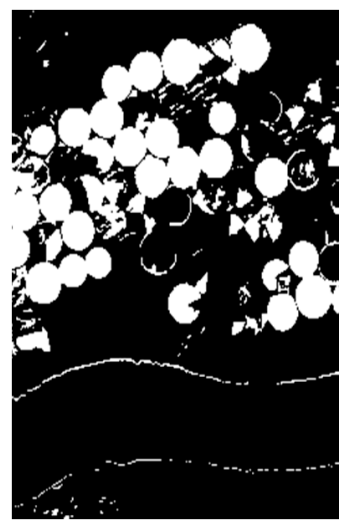

(c)

Figure 1. False color composites of the original hyperspectral images acquired in (a) 2004 and (b) 2007 of the USA data set, and (c) binary change map ground truth.

Due to environmental and equipment conditions, data pre-processing plays a crucial role in RS before the beginning of the main process. The pre-processing stage is made up of two steps: (1) geometric preprocessing and (2) spectral preprocessing (including remove no-data bands, destriping, noise reduction, smile-frown detection, radiometric calibration, and atmospheric correction) [7].

\subsection{Adopted Methodology}

The focus of this research is to produce the probability map of occurrence of changes in the image scene. To this end, distinguishing changed and no-changed pixels is crucial. In the past decades, many binary $\mathrm{CD}$ algorithms have been proposed. After discriminating changed and no-changed pixels, the changed ones will be categorized as a percentage of probabilities based on the proposed approach. According to the flowchart in Figure 1, the proposed framework consists of the following four steps after pre-processing. In the first step, a binary change map (BCM) is produced and changed/no-changed pixels are discriminated. In the second step, an unmixing process is utilized. In order to produce a 
probability map of changes between two bi-temporal images, some similarity metrics are used in the third step for measuring the spectral similarity of pixels in the abundance maps of endmembers. A measurement of spectral similarity identifies the finer spectral differences between the two HSIs. Finally, in the last step the spectral similarity values are masked by the BCM and a probability map is produced.

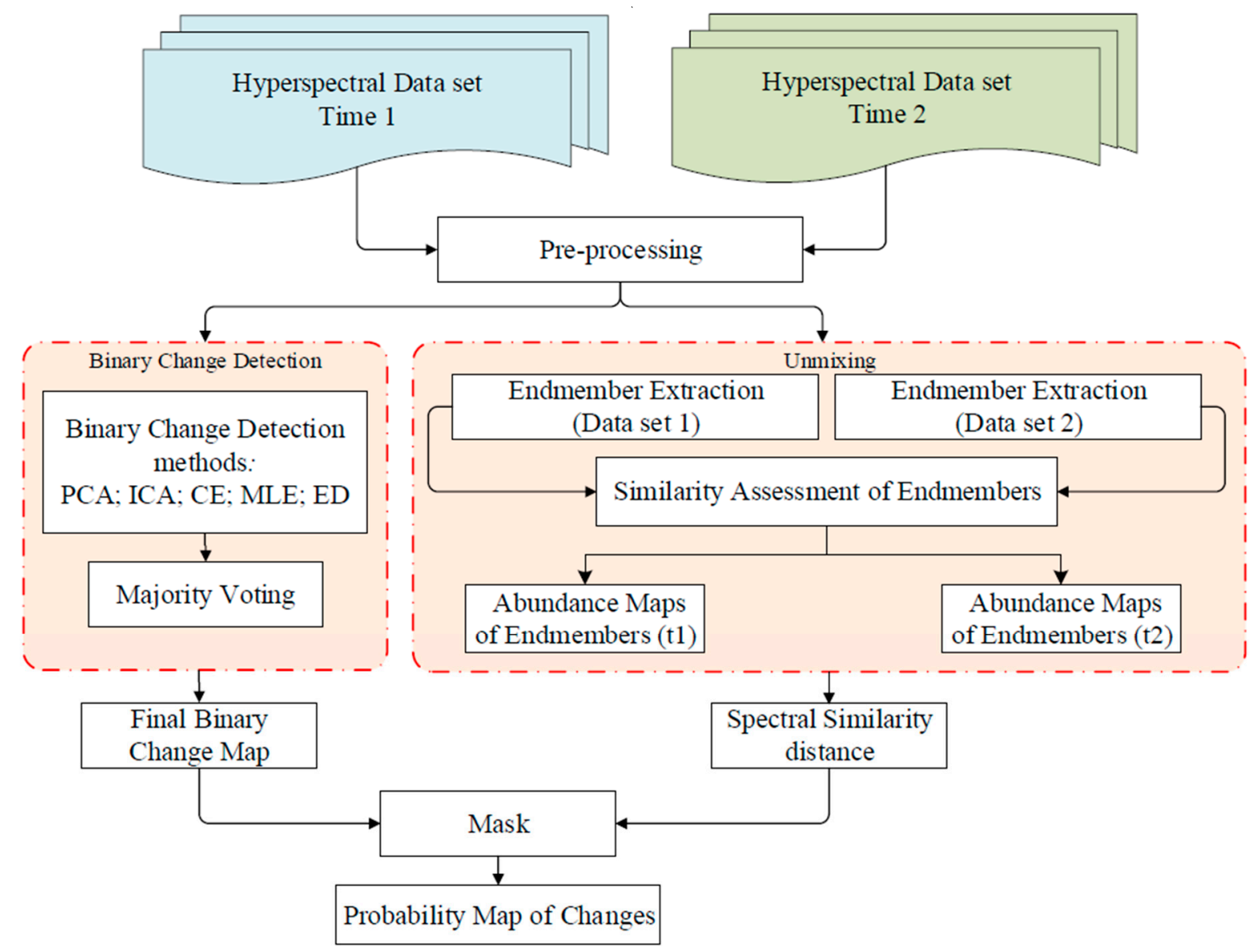

Figure 2. The flowchart of the proposed approach to produce probability map of changes. PCA: principal component analysis; ICA: independent component analysis; CE: cross equalization; MLE: maximum likelihood estimator; ED: Euclidean distance.

\subsubsection{Step 1}

In order to distinguish changed and no-change pixels, we used five binary CD methods that have been previously proposed and widely used in the literature. The most representative changed/nochanged methods are based on arithmetic operations and transformations, such as principal component analysis (PCA), independent component analysis (ICA), cross equalization (CE), maximum likelihood estimator (MLE), and Euclidian distance (ED) [6]. Figure 3 depicts the visual analysis of the results of aforementioned binary CD methods. The final BCM was obtained using the majority voting concept (of implemented methods) to achieve a change map with high accuracy. 


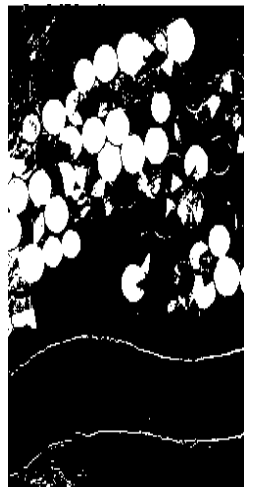

(a)

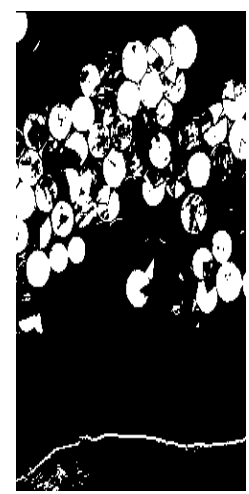

(b)

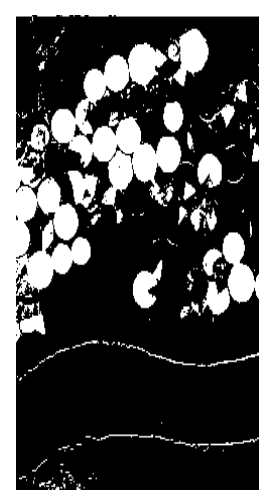

(c)

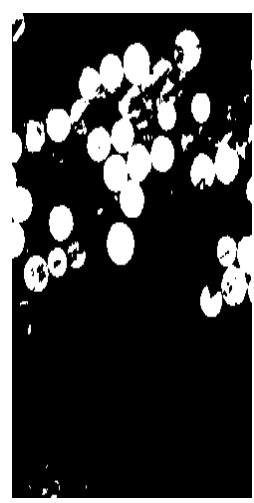

(d)

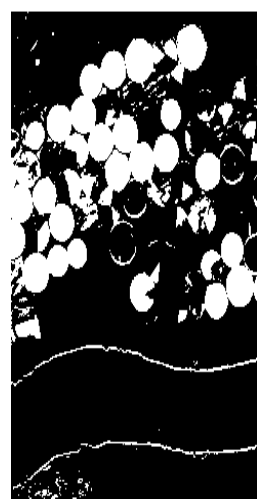

(e)

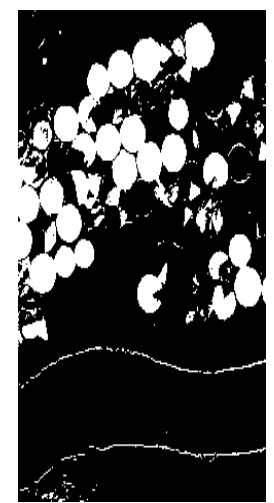

(f)

Figure 3. Result of the performance of binary change detection methods in USA data set. (a) PCA, (b) ICA, (c) CE, (d) MLE, (e) ED, and (f) final BCM.

A numerical evaluation of the implemented methods and final BCM is presented in Table 1 . The accuracy of the CD methods is measured through overall accuracy (OA), kappa coefficient $(\kappa)$, false positive rate (FPR), and the Matthews correlation coefficient (MCC) indices.

Table 1. Numerical analysis of binary change detection methods. OA: overall accuracy; $\kappa$ : kappa; FPR: false positive rate; MCC: Matthews correlation coefficient.

\begin{tabular}{ccccc}
\hline Method & OA (\%) & $\boldsymbol{\kappa}$ & FPR & MCC \\
\hline PCA & 96.20 & 0.8835 & 0.0583 & 0.8845 \\
ICA & 88.68 & 0.6504 & 0.2425 & 0.6516 \\
CE & 96.54 & 0.8909 & 0.0672 & 0.8912 \\
MLE & 87.83 & 0.5874 & 0.3856 & 0.5900 \\
ED & 96.15 & 0.8852 & 0 & 0.8911 \\
Final BCM & 97.00 & 0.9055 & 0.0527 & 0.9059 \\
\hline
\end{tabular}

\subsubsection{Step 2}

Endmember extraction is a vital step in the spectral unmixing of HSIs. Endmembers refer to the pure materials spectra in HSIs, and endmember extraction is a process of finding the spectra of all the endmembers [7]. In this study, the endmembers were extracted using the simplex identification via split augmented Lagrangian (SISAL) algorithm [8]. Then, similarity assessment of endmembers based on spectral angle measure (SAM) technique was adopted (see Equation (1)). Finally, the fully constrained least squares (FCLS) method proposed in Heinz et al. [9] was used to estimate the fraction of abundances.

\subsubsection{Step 3}

The objective of this step is to make an analysis of four norm similarity metrics in order to produce changes' probability maps. The selection of these metrics was conducted considering its aim and good performance of detecting spectral differences. According the above mentioned principle, the selected metrics were SAM [10], Pearson correlation coefficient (PCC) [11], Bray-Curtis dissimilarity (BCD) [12], and Jeffries-Matusita distance (JMD) [13]. These metrics will be explained briefly in the following.

The SAM technique was proposed in Kruse et al. [10] to measure the similarity between two spectral feature vectors. In Renza et al. [14], the angle between each multi-temporal image and a reference vector measured by means of a SAM metric was proposed as a solution for CD in specific land covers. In this paper, the spectral similarity was based on SAM obtained by considering each 
spectrum as a vector in the abundance maps of endmembers. This algorithm can be calculated as Equation (1) [14-16].

$$
\mathrm{S}_{\alpha}=\cos ^{-1}\left(\frac{\sum_{\mathrm{i}=1}^{\mathrm{n}} \mathrm{x}_{\mathrm{i}} \mathrm{y}_{\mathrm{i}}}{\left|\sum_{\mathrm{i}=1}^{\mathrm{n}} \mathrm{x}_{\mathrm{i}}^{2}\right|^{\frac{1}{2}}\left|\sum_{\mathrm{i}=1}^{\mathrm{n}} \mathrm{y}_{\mathrm{i}}^{2}\right|^{\frac{1}{2}}}\right)
$$

where $x$ is the spectral signature vector of a pixel (in the abundance maps of endmembers) in the image of time $t_{1}, y$ is the spectral signature vector of a pixel (in the abundance maps of endmembers) in the image of time $t_{2}$, and $n$ is the number of endmembers. The spectral angle goes from 0 when signatures are identical to 90 when signatures are completely different.

The PCC is one of the most popular measures for calculating the dependency between two spectral vectors [11]. The PCC between spectral random vectors is defined as Equation (2) [11]. This measure is widely used in RS applications. A correlation of -1.0 shows a perfect negative correlation, while a correlation of 1.0 shows a perfect positive correlation. A correlation of 0.0 shows no relationship between the movements of the two variables.

$$
P_{x y}=\frac{\sum_{i=1}^{n}\left(x_{i}-\bar{x}\right)-\left(y_{i}-\bar{y}\right)}{\sqrt{\sum_{i=1}^{n}\left(x_{i}-\bar{x}\right)^{2}} \sqrt{\sum_{i=1}^{n}\left(y_{i}-\bar{y}\right)^{2}}}
$$

The BCD metric is one of the most well-known dissimilarity metrics for quantifying differences between samples. This measure appears to be very reasonable way of achieving this goal and takes on values between 0.0 (when signatures are completely different) and 1.0 (when signatures are identical) [12]. The normalized formula for calculating the BCD between two samples is given as Equation (3) [12].

$$
\mathrm{S}(\mathrm{x}, \mathrm{y})=\frac{\sum_{\mathrm{i}=1}^{\mathrm{n}}\left|\mathrm{x}_{\mathrm{i}}+\mathrm{y}_{\mathrm{i}}\right|}{\sum_{\mathrm{i}=1}^{\mathrm{n}}\left(\mathrm{x}_{\mathrm{i}}-\mathrm{y}_{\mathrm{i}}\right)}
$$

The JMD is a widely used statistical separability criterion. It is a parametric criterion, for which the values range between 0.0 (when signatures are identical) and 2.0 (when signatures are completely different). The JMD is calculated as Equation (4) [13].

$$
\mathrm{J}_{\mathrm{xy}}=2\left(1-\mathrm{e}^{-\mathrm{B}}\right)
$$

where

$$
\mathrm{B}=\frac{1}{8}(\mathrm{x}-\mathrm{y})^{\mathrm{T}}\left(\frac{\sum \mathrm{x}+\sum \mathrm{y}}{2}\right)^{-1}(\mathrm{x}-\mathrm{y})+\frac{1}{2} \ln \left(\frac{\left|\frac{\sum \mathrm{x}+\sum \mathrm{y}}{2}\right|}{\left.\left|\sum \mathrm{x}\right|^{\frac{1}{2}}|| \sum \mathrm{y}\right|^{\frac{1}{2} \mid}}\right)
$$

\subsubsection{Step 4}

In the last step of the methodology, the results of step 3 are masked by the final BCM produced in step 1 . For achieving the final probability map of changes, we utilized majority voting concept.

\section{Results and Discussion}

Based on aforementioned spectral similarity metrics, probability maps of changes produced and the results are shown in Figure 4. According to the obtained results, the probability maps produced by PCC and BCD methods are similar to each other (see Figure $4 b, c)$. 


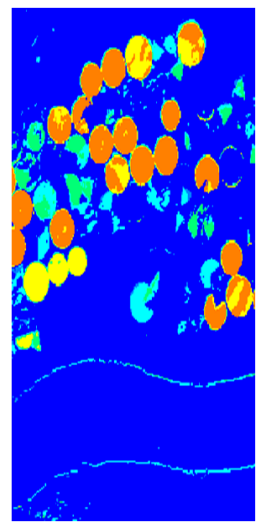

(a)

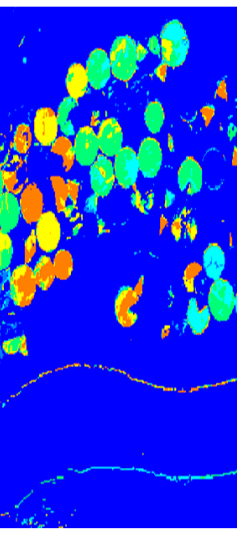

(b)

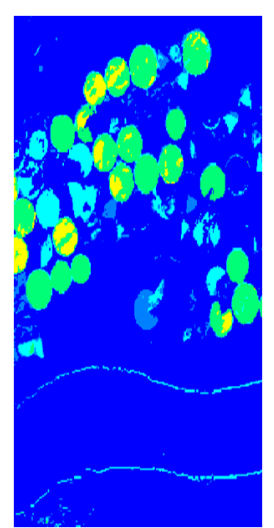

(c)

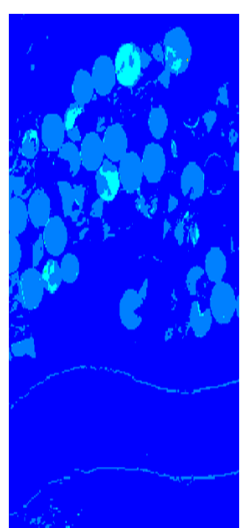

(d)
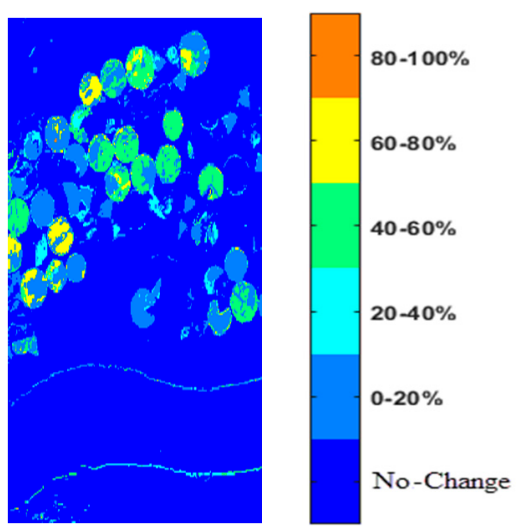

(e)

(f)

Figure 4. Results of the estimated probability maps of employed spectral similarity metrics. (a) SAM, (b) PCC, (c) BCD, (d) JMD, (e) final probability map of changes, and (f) legend.

By utilizing majority voting, the probability map of changes is produced more confidently. In this study, the probability analysis was applied to explore changed pixels. as shown in Figure $4 \mathrm{f}$, the probability of changes is expressed in percentage form (on a scale from $0 \%$ to 100\%). Figure $4 \mathrm{e}$ illustrates that the probability of most of the changed pixels is in the range of $40-60 \%$. The aim of experimental evaluation is to prove the efficiency of the proposed methodology based on similarity metrics, for representation of changes' probability.

\section{Conclusions}

CD plays a key role in many applications, including assessing natural disasters, monitoring crops, and managing water resources. In this study, an approach for analyzing detected changes was proposed. Utilizing the nature of occurred changes along with the probability of changes is a strategy that can be applied for better management of a study area. This methodology can be investigated and discussed in details for data sets with available probability map of changes.

Author Contributions: Formal analysis and methodology, H.J. and M.H.; Writing-original draft, H.J.; Writing-review and editing, H.J. and M.H.

Conflicts of Interest: The authors declare no conflict of interest.

\section{Abbreviations}

The following abbreviations are used in this manuscript:
RS: remote sensing
HSI: hyperspectral image
CD: change detection
BCM: binary change map
SAM: spectral angle measure
PCC: Pearson correlation coefficient
BCD: Bray-Curtis dissimilarity
JMD: Jeffries-Matusita distance

\section{References}

1. Sadeghi, V.; Ahmadi, F.F.; Ebadi, H. A new fuzzy measurement approach for automatic change detection using remotely sensed images. Meas. 2018, 127, 1-14. 
2. Liu, S.;Bruzzone, L.;Bovolo, F.; Du, P. Unsupervised Multitemporal Spectral Unmixing for Detecting Multiple Changes in Hyperspectral Images.IEEE Transactions on Geoscience Remote Sensing, 2016. 54(5): pp. 2733-2748.

3. Meola, J.;Eismann, M.T.;Moses, R.L.; Ash, J.N. Detecting Changes in Hyperspectral Imagery Using a Model-Based Approach.IEEE transactions on geoscience remote sensing, 2011. 49(7): pp. 2647-2661.

4. Shah-Hosseini, R.; Homayouni, S.; Safari, A. Environmental monitoring based on automatic change detection from remotely sensed data: kernel-based approach. J. Appl. Remote. Sens. 2015, 9, 95992.

5. Singh, A. Review Article Digital Change Detection Techniques Using Remotely-Sensed Data.International journal of remote sensing, 1989. 10(6): pp. 989-1003.

6. Hasanlou, M.;Seydi, S.T. Hyperspectral Change Detection: An Experimental Comparative Study.International journal of remote sensing, 2018. 39(20): pp. 7029-7083.

7. Ertürk, A.;Plaza, A. Informative Change Detection by Unmixing for Hyperspectral Images.IEEE Geoscience Remote Sensing Letters, 2015. 12(6): pp. 1252-1256.

8. Bioucas-Dias, J. A variable splitting augmented Lagrangian approach to linear spectral unmixing. 2009 First Workshop on Hyperspectral Image and Signal Processing: Evolution in Remote Sensing 2009.

9. Heinz, D.;Chang, C.-I.; Althouse, M.L. "Fully constrained least-squares based linear unmixing [hyperspectral image classification]." IEEE 1999 International Geoscience and Remote Sensing Symposium. IGARSS'99 (Cat. No. 99CH36293). Vol. 2. IEEE, 1999

10. Kruse, F.A.; Lefkoff, A.B.; Boardman, J.W.; Heidebrecht, K.B.; Shapiro, A.T.; Barloon, P.J.; Goetz, A.F.H. The spectral image processing system (SIPS)-interactive visualization and analysis of imaging spectrometer data. AIP Conference Proceedings 1993, 283, 192-201.

11. Coefficient, W.J.P.C., Encyclopedia of Systems Biology. 2013, Springer, New York, NY, USA.

12. Clarke, K.R.; Somerfield, P.J.; Chapman, M.G. On resemblance measures for ecological studies, including taxonomic dissimilarities and a zero-adjusted Bray-Curtis coefficient for denuded assemblages. J. Exp. Mar. Boil. Ecol. 2006, 330, 55-80.

13. Dabboor, M.; Howell, S.; Shokr, M.; Yackel, J. The Jeffries-Matusita Distance for the Case of Complex Wishart Distribution as a Separability Criterion for Fully Polarimetric Sar Data. Int. J. Remote. Sens., 2014. 35(19): pp. 6859-6873.

14. Renza, D.; Martinez, E.; Molina, I.; L., D.M.B. Unsupervised change detection in a particular vegetation land cover type using spectral angle mapper. Adv. Space Res. 2017, 59, 2019-2031.

15. Júnior, O.A.C.; Guimarães, R.F.; Gillespie, A.R.; Silva, N.C.; Gomes, R.A.T. A New Approach to Change Vector Analysis Using Distance and Similarity Measures. Remote. Sens. 2011, 3, 2473-2493.

16. Ramos, J.F.; Renza, D.; L., D.M.B. Evaluation of spectral similarity indices in unsupervised change detection approaches. DYNA 2018, 85, 117-126.

(C) 2019 by the authors; licensee MDPI, Basel, Switzerland. This article is an open access article distributed under the terms and conditions of the Creative Commons Attribution (CC-BY) license (http://creativecommons.org/licenses/by/4.0/). 